\title{
Supramolecular Fibers In Gels Can Be At Thermodynamic Equilibrium: A Simple Packing Model Reveals Preferential Fi- bril Formation Versus Crystallization
}

\author{
Ivan Ramos Sasselli, ${ }^{\dagger}$ Peter J. Halling, ${ }^{\dagger}$ Rein V. Ulijn, ${ }^{*,+,}$ and Tell Tuttle*,† \\ ${ }^{\dagger}$ Pure and Applied Chemistry Department, WestCHEM, University of Strathclyde, 295 Cathedral Street, Glasgow, G1 1XL, \\ UK. \\ ${ }^{\ddagger}$ Advanced Science Research Center (ASRC) and Hunter College, City University of New York, 85 St Nicholas Terrace, \\ New York, NY 10031, USA.
}

KEYWORDS Low molecular weight gelators, amphiphiles, soft-matter, gel, self-assembly, thermodynamics, packing, model.

\begin{abstract}
Low Molecular Weight Gelators are able to form nanostructures, typically fibers, which entangle to form gel-phase materials. These materials have wide-ranging applications in biomedicine and nanotechnology. While it is known that supramolecular gels often represent metastable structures due to the restricted molecular dynamics in the gel state, the thermodynamic nature of the nanofibrous structure is not well understood. Clearly, 3D extended structures will be able to form more interactions than 1D structures. However, self-assembling molecules are typically amphiphilic, thus giving rise to a combination of solvophobic and solvophilic moieties where a level of solvent exposure at the nanostructure surface is favorable. In this study, we introduce a simple packing model, based on prisms with faces of different nature (solvophobic and solvophilic) and variable interaction parameters, to represent amphiphile self-assembly. This model demonstrates that by tuning shape and 'self' or 'solvent' interaction parameters either the 1D fiber or 3D crystal may represent the thermodynamic minimum. The model depends on parameters that relate to features of experimentally known systems: the number of faces exposed to the solvent or buried in the fiber; the overall shape of the prism; and the free energy penalties associated with the interactions can be adjusted to match their chemical nature. The model is applied to describe the $\mathrm{pH}$ dependent gelation/precipitation of well-known gelator Fmoc-FF. We conclude that, despite the fact that most experimentally produced gels probably represent metastable states, one-dimensional fibers can represent thermodynamic equilibrium. This conclusion has critical implications for the theoretical treatment of gels.
\end{abstract}

A wide range of small molecules which are able to selfassemble into supramolecular gels have been investigated over the years as promising new materials with potential applications in nanotechnology and biomedicine..$^{1-17}$ Low Molecular Weight Gelators (LMWGs), spontaneously form nano- or micro-structures in solutions to create a solvent supporting network. LMWGs are highly variable in their molecular composition and include peptides, ${ }^{17-18}$ sugar derivatives, ${ }^{19-20}$ and other amphiphiles, ${ }^{15,21}$ offering opportunities for the assembly of materials with broad ranging properties in a simple bottomup approach. In these systems, the functionality is dictated by supramolecular structure and the structure can in turn be tuned by changing the self-assembling unit as well as the selfassembly pathway. ${ }^{14-15,21-26}$ This pathway dependence clearly suggests that there are both thermodynamic and kinetic aspects to gel formation and gel structure. Indeed, metastable gels, obtained by controlling the pathway and kinetics of the gelation, have resulted in materials with different nanostructures and properties. ${ }^{27-34}$ In some cases, different kinetically trapped gels could eventually evolve into the same gel state by using elevated temperatures to overcome kinetic barriers, which suggests that gels may represent thermodynamic minima that can be accessed reversibly. ${ }^{27-29}$

LMWGs are usually amphiphilic in nature. As such, solvophobic parts of the molecules have a tendency to aggregate while solvophilic parts are preferentially solvent-exposed, thus giving rise to the formation of supramolecular nanostructures. It is clear that not all amphiphiles are able to gelate and there must be a balance between the solvophobic and solvophilic parts to allow the formation of the nanostructures that remain stabilized within the solvent environment. When this balance is not met, the molecule may either be too solvophilic, resulting in the molecule not aggregating, or forming small aggregates such as micelles that remain in solution, while when it is too solvophobic it may precipitate or crystallize. Therefore, the gelation tendency is observed for molecules with a limited solubility in a given solvent. ${ }^{27,35-38}$

For the thermodynamic nature of the gel state, two different scenarios have been proposed. In the first scenario, the gel state (GS) is a kinetically trapped metastable state, which avoids the transformation of the system to the crystals state (CS). ${ }^{16,36-42}$ The main argument supporting this is that a $3 \mathrm{D}$ extended structure (CS) allows a higher number of stabilizing interactions than a $1 \mathrm{D}$ structure (GS). Depending on the depth of the metastable state and the activation barriers around it, the system could transition to the CS and this gel-crystal transition has been observed for several systems. ${ }^{37-38,40-45}$

The alternative scenario is that for certain gelators the $1 \mathrm{D}$ structures formed in the GS represent the thermodynamic minimum. For some examples which have clearly defined solvophobic and solvophilic sections, preferential formation of 1D structures, akin to worm-like micelles, is quite intuitive, 
e.g., Stupp's peptide amphiphiles. ${ }^{9}$ The concept of thermodynamically favored formation of $1 \mathrm{D}$ nanostructures has been successfully applied in molecular dynamic simulations and in studies of gels to develop tools to investigate the gelation tendencies as a function of the free energy. ${ }^{18,46}$ Furthermore, self-healing gels depend on the possibility of gels to represent the thermodynamic equilibrium (although it could be argued that a local minimum could also represent a gel state that can be reversible accessed). It has been argued that "thermodynamically stable gels" represent deep local minima surrounded by high activation barriers which may make it impossible to access the global minimum, the CS. ${ }^{36-37,39-42}$

Simple models have previously been used to describe gelation and crystallization behavior of proteins. Dixit et al. developed a theoretical model to rationalize the gel-crystal equilibrium for proteins. ${ }^{47}$ This model is based on the idea that only in the CS the molecules are organized by well-ordered packing while the GS is an amorphous disordered state. However, it is known that the GS, in the case of most LMWGs, is actually the result of extended well-ordered interactions. Therefore, this kinetic model is not necessarily applicable for LMWGs.

The problem in the case of LMWGs is further complicated due to the similarity and degree of order of the molecular packing in both the CS and the GS, which has been observed experimentally. ${ }^{37-38,41-42,45}$ The shape is critical to represent LMWGs and although they have been represented by spheres before, ${ }^{18,48}$ these procedures considered each molecule represented by a number of beads rather than representing each molecule by only one unit. LMWGs are not reasonably represented by spheres because with a spherical potential in the 3D extended structure the CS will always be the most stable. A model for these molecules should take into account the amphiphilicity of the LMWG, which is clearly critical to formation of 1D fibers and gelation. Therefore, the challenge is to develop a simple packing model, which takes into account the amphiphilicity of the units, to determine whether fibers, representing the GS, are able to provide a thermodynamic minimum that is more stable than the CS.

In this paper we introduce a model that represents the selfassembling molecules as prisms which present faces of different nature: solvophobic; and solvophilic. The concept of faces of different nature depending on the chemical groups present on them is commonly applied in crystallography and models have been developed to rationalize the final shapes of crystals. ${ }^{49-50}$ However, these are kinetic models which deal with the different growth rates on the different faces of crystals. In contrast, our model focuses on the potential equilibrium stability of fibers of differing lateral dimension (based in the individual molecular units) with reference to the crystals (vida infra).

This model shows how the amphiphilicity of the assembling units introduce the possibility of achieving a thermodynamic minimum with a $1 \mathrm{D}$ infinite structure rather than a $3 \mathrm{D}$ one, demonstrating that fibers can represent the thermodynamically favored assembled form for certain LMWGs.

The model is developed for an isolated fiber as a precondition for the formation of gels as the thermodynamic minimum state (but is also generally applicable for selfassembling molecules that form 1D structures, not all of which will form gels). The gel will result from the entanglement of the fibers to create a nanofibrous network. If the network involves only a minimal contact surface between fibers the interactions between the fibers can, as a first approximation, be considered negligible in comparison with the whole fiber surface. Therefore, while the model considers only the thermodynamics of the initial fiber formation this step is the critical factor in determining whether the resulting gel will be at the thermodynamic minimum, relative to the crystal state. However, if the network involves significant overlap between the fiber surfaces then this approximation is no longer valid and the network represents a metastable state that tends towards the crystal state.

Our model is in good agreement with both proposed theories, as it shows that the GS or CS can represent the thermodynamic minimum depending on key characteristics of the system related to balance of solvophobicity and solvophilicity of the LMWG in a solvent. Finally, the model is extended to other geometries to show its validity and applicability to known experimental examples. We demonstrate that the model can be used to describe the behavior of aromatic peptide amphiphiles under changing $\mathrm{pH}$ conditions. ${ }^{51}$

\section{USING PRISMS TO REPRESENT LMWG}

The amphiphilicity of LMWGs is clearly a key property that allows the molecules to self-assemble into nanostructures. The presence of "regions" of different nature - solvophobic and solvophilic - allows these molecules to adopt orientations based on a balance between the interactions with the solvent and interactions with other solute molecules. Here, we describe the use of square based prisms $(\mathrm{Sq})$, cubes, to represent the amphiphilicity of the self-assembling units by considering equal sized faces of different nature (Fig. 1). However, the model can be readily extended to other regular shaped prisms (Fig. S1-S5). The model considers the fiber to be of infinite length in the $z$-direction with the base of the prisms in the $x y$ plane, the cross-section of the fiber. The width of the fiber is a function of the parameter $\mathrm{d}$, which is defined as the number of units per side (Fig. 1).

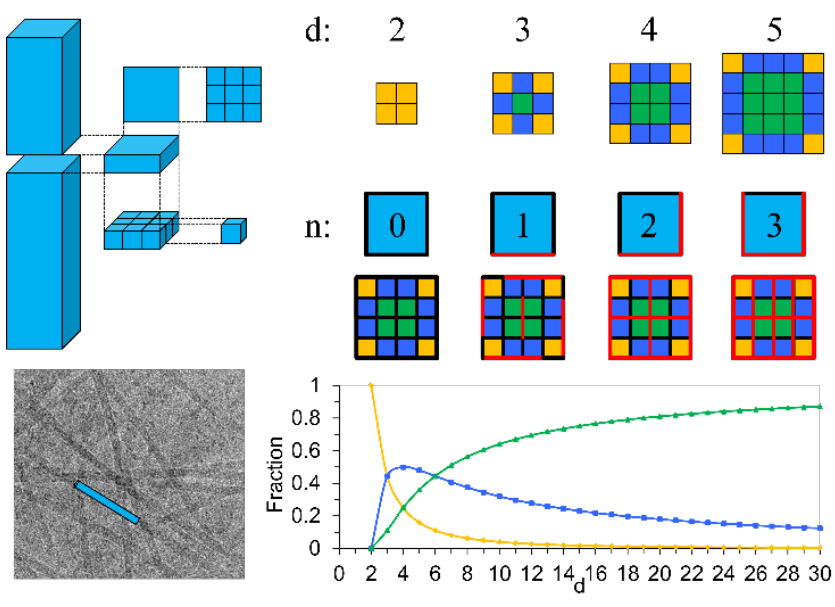

Figure 1. Schematic of the model from fibers to the molecular unit (Left), and the cross-section as a function of $n$ (the number of solvophilic faces in the unit) and as a function of $d$ (the number of units along one side of the fibre, Right). Solvophilic faces are shown in red and solvophobic in black. Lines inside of the fiber are two faces of the same nature, solvophobic or solvophilic, opposed to form the most stable structure. The graph shows the evolution of the fractions of the different units as a function of $d$. Yellow represents corner units (two faces exposed to solvent), 
blue the sides (one face exposed) and green the fraction of unexposed units.

\section{FORMULATION}

The model accounts for the Gibbs energy as the sum of the contributions of the different faces of the prisms that make up the fiber. Clearly, neither the entropic (e.g., the immobilization of the molecules into the fiber, the release of solvent, etc.) nor enthalpic (e.g., H-bonding, $\pi$-stacking, etc.) contributions are strictly additive. However, it is common to approximate them as such. For example, the estimation of solvation Gibbs energies in terms of surface area contributions, common with proteins, uses the additivity of entropic contributions and cooperative effects within H-bonding are well known. ${ }^{52}$ However, this is a relatively minor approximation within our mesoscale model and should not affect the validity of the general results that the model achieves.

There are two different types of faces: solvophilic $(l$, red in Fig. 1) and solvophobic ( $k$, black in Fig. 1), and these faces can be either exposed to the solvent $(s)$ or buried inside the fiber $(b)$. Hence, there are four possible interactions and their contributions to the Gibbs energy are $\gamma_{l s}, \gamma_{l b}, \gamma_{k s}$ and $\gamma_{k b}$.

The magnitude, and relative ordering of these parameters is clearly system dependent and different examples are provided below. The favorability of the formation of fibers over crystals requires the interactions between the solvent and exposed faces of the LMWG to be more favorable than the solventsolvent interactions. While if the solvent-solvent interaction is more favorable, crystals will always be preferred as they eliminate solvent-monomer interactions at the limit of infinite size. Therefore, the relative stability of the solvent-solvent and solvent-fiber interactions is considered within the model with the election of the values used for the $\gamma_{k s}$ and $\gamma_{l s}$ parameters. In addition, the model also depends on the solvophobic interactions through the parameter $\gamma_{k b}$, which is fundamentally the result of solvent-solvent interactions. However, this dependence does not stop the interaction being well described in terms of Gibbs energy contributions when hydrophobic surfaces contact each other, rather than contacting water. Therefore, the relative stability of the solvent-solvent and solventfiber interactions is considered within the model with the election of the values used for the $\gamma_{k s}$ and $\gamma_{l s}$ parameters. These parameters do not consider only the direct interaction of the solvent with the self-assembling molecule but also the effect on the solvent, as it is considered implicitly.

However, to calculate the total contribution to the Gibbs energy of the fiber $\left(G_{f i b e r}\right)$, these individual contributions are multiplied by the fraction of faces $\left(f_{l s}, f_{l b}, f_{k s}\right.$ and $\left.f_{k b}\right)$ involved in the different interactions (Eq. 1). By using fractions of the different types of units rather than absolute numbers of units, it is straightforward to consider infinitely large systems in the comparison between fibers and crystals. This is not possible in standard computational methods, which contain fixed number of molecules.

$$
\begin{gathered}
G_{\text {fibre }}=f_{l s} \cdot \gamma_{l s}+f_{l b} \cdot \gamma_{l b}+f_{k s} \cdot \gamma_{k s}+f_{k b} \\
\cdot \gamma_{k b} \quad(1)
\end{gathered}
$$

These parameters can be related with the total contribution to the Gibbs energy of the crystal state $\left(G_{c r y s}\right)$ and the solution state $\left(G_{\text {solv }}\right)$ :

$$
G_{c r y s}=f_{l b} \cdot \gamma_{l b}+f_{k b} \cdot \gamma_{k b}
$$

$$
G_{\text {solv }}=f_{l s} \cdot \gamma_{l s}+f_{k s} \cdot \gamma_{k s}
$$

\section{PARAMETERS DEFINITION}

The model derives its parameters from rational considerations:

$n \leq m_{\max }$ : The number of solvophilic faces in the unit $(n)$ cannot be higher than the maximum number of solvent exposed faces in the cross-section $\left(m_{\max }\right)$. For example, $m_{\max }$ of square prisms is 2 and it already covers the whole fiber surface with solvophilic faces, which has a stabilizing effect (Fig. 1). However, when the units have one more solvophilic face $(n=3)$, the extra faces are buried, which has a destabilizing effect, adding no stabilizing effect to compensate it. This also limits the solubility of the molecules considered in this theory and gives rise to two types of fibers:

- $n<m_{\max }$ : the fiber exposes both, solvophilic and solvophobic faces to the solvent; i.e. the fiber surface has heterogeneous faces and therefore has an amphiphilic character.

- $n=m_{\max }$ : the fiber shows a homogenously solvophilic surface but as a result will have solvophilic faces buried in the core for $d>2$ (Fig. 1).

$n=0$ is included as a non-amphiphilic reference and $n=3$ is included to show the effect of having $n>m_{\max }$.

$G_{\text {crys }}<G_{\text {solv }}$ : This requirement excludes molecules that are too well solvated to form crystals or fibers.

\section{MODEL DEVELOPMENT}

First, we derive a quantitative formula to describe how exposure of unit faces changes with increased width of a model fiber $(d)$. The fractions of exposed faces are calculated by dividing the number of units with $m$ faces exposed to the solvent in the cross-section $\left(N_{m}\right)$ by the total number of units on the cross-section $\left(N_{\text {tot }}\right)$, giving rise to parameters $f_{m}$ (Eq.4). Square prisms can have zero (buried, $f_{0}$, shown in green), one (fiber side, $f_{1}$, blue) or two faces (corner of the fiber, $f_{2}$, yellow) exposed to the solvent (Fig. 1). Both parameters are a function of the parameter $d$.

$$
f_{m}=\frac{N_{m}(d)}{N_{t o t}(d)}
$$

The $f_{m}$ plots start at $d=2$ because $d=1$ would require a different formulation to represent all the faces in the crosssection exposed to the solvent (Fig. 1). In the fraction plot it can be seen how $f_{2}$ (the contribution of the corners) is only important at small values of $d$ and decreases rapidly with increasing fiber width (Fig. 1). Also $f_{l}$ (the sides of the fiber) contributes at small values of $d$ (except for the minimum width where the fiber has no side units) and its contribution decreases more slowly, relative to $f_{2}$. The contribution of $f_{0}$ increases with $d$ and it tends to 1 at infinite values of the $d$. While the exact interplay of the different parameters is described in detail below, this graph already suggests the possibility of balanced interactions at low $d$, which would correspond to fiber-like structures. The equations for $f_{2}, f_{l}$ and $f_{0}$ can be found in Table S3.

To calculate the $G_{\text {fiber }}$ it is necessary to consider the relationship between $f_{m}$ and the fraction of faces involving the different interactions $\left(f_{\text {int }}\right)$. The relationship between these parameters depends on the geometry of the units and on the number 
of solvophilic faces. The general relationship between these two parameters is described with the equation:

$$
f_{\text {int }}=\sum_{m=0}^{2} f_{m} \alpha_{i n t, m}
$$

Where the factor $\alpha_{i n t, m}$ represents the number of faces that units with $m$ faces exposed to the solvent have involving the different interactions $(l s, l b, k s$ or $k b)$. Therefore the general equation 1 is now:

$$
\begin{aligned}
G_{\text {fiber }}=\sum_{m=0}^{2}( & \left.f_{m} \alpha_{l s, m}\right) \gamma_{l s}+\sum_{m=0}^{2}\left(f_{m} \alpha_{l b, m}\right) \gamma_{l b} \\
& +\sum_{m=0}^{2}\left(f_{m} \alpha_{k s, m}\right) \gamma_{k s} \\
& +\sum_{m=0}^{2}\left(f_{m} \alpha_{k b, m}\right) \gamma_{k b}
\end{aligned}
$$

The factors $\alpha_{i n t, m}$ can be determined for the case of squares with $n=1\left(S q_{1}\right)$ by looking at the disposition of the units in the structure in Fig. $1\left(\alpha_{i n t, m}\right.$ values not mentioned are 0$)$ :

int $=l s$ : Units with $m=2$ and with $m=1$ expose one solvophilic face to the solvent, therefore: $\alpha_{l s, 2}=1$ and $\alpha_{l s, 1}=1$.

int $=l b$ : Only units with $m=0$ bury their solvophilic face inside of the fiber: $\alpha_{l b, 0}=1$.

int $=k s$ : Only units with $m=2$ expose one solvophobic face to the solvent: $\alpha_{k s, 2}=1$.

int $=k b$ : Units with $m=2$ bury 4 solvophobic faces, while $m=1$ and $m=0$ bury 5 each: $\alpha_{k b, 2}=4, \alpha_{k b, 1}=5$ and $\alpha_{k b, 0}=5$.

The resulting equation is:

$$
\begin{aligned}
G_{\text {fiber }}\left(S q_{1}\right)= & \left(f_{2}+f_{1}\right) \gamma_{l s}+f_{0} \gamma_{l b}+f_{2} \gamma_{k s} \\
& +\left(4 f_{2}+5 f_{1}+5 f_{0}\right) \gamma_{k b}
\end{aligned}
$$

Related equations can be developed for other regular shaped prisms (Table S4). For ease of comparison between the competing states, the results are presented using the $\Delta G_{\text {fiber }}$ :

$$
\Delta G_{\text {fiber }}=G_{\text {fiber }}-G_{\text {crys }}
$$

In this way the results can be interpreted:

$\Delta G_{\text {fiber }}<0 ; G_{\text {fiber }}<G_{\text {crys. }}:$ The fiber represents the thermodynamic minimum.

$\Delta G_{\text {fiber }}>0 ; G_{\text {fiber }}>G_{\text {crys }}:$ The crystal represents the thermodynamic minimum.

The proposed formulation is applied to derive the $\Delta G_{\text {fiber }}$ expressions for $S q$ with 0 to 3 solvophilic faces (Table S1). $n=0$ corresponds to a non-amphiphilic molecule; and $n=3$ is higher than the $n_{\max }$ proposed $\left(n_{\max }=m_{\max }=2\right)$ in the parameters definition section. These are used as controls to show how amphiphilicity is required to have fibers at thermodynamic equilibrium.

Finally, taking into account that in solvation all the faces are exposed $\left(f_{6}=1\right)$ and in the crystal all are buried $\left(f_{0}=1\right)$ and applying equation 5 to equations 2 and 3 , the relationship between the parameters for the different types of interactions $\left(\gamma_{i}\right)$ and of the crystal Gibbs energy $\left(G_{c r y s}\right)$ and of solvation excess Gibbs energy $\left(G_{\text {solv }}\right)$ can be obtained (Table S2). The resulting equations for $S q_{n}$ are:

$$
\begin{aligned}
& G_{c r y s}=n \gamma_{l b}+(6-n) \gamma_{k b} \\
& G_{s o l v}=n \gamma_{l s}+(6-n) \gamma_{k s}
\end{aligned}
$$

Minimum values for $G_{c r y s}$ and $G_{\text {solv }}$ were calculated (Table S2) using the minimum values for $\gamma_{l b}$ and $\gamma_{k s}$ mentioned below, i.e. 0 and 2 respectively. The equations are applied from these minimum values and as well as a function of $d$ they are presented as a function of the parameters $\gamma_{l b}$ or $\gamma_{k s}$ (and hence of $G_{\text {crys }}$ or $G_{\text {solv }}$ ) to show the effect in the $\Delta G_{\text {fiber }}$ of increasing the destabilizing effect of these interactions. The parameters $\gamma_{l b}$ and $\gamma_{k s}$, can be related with the interactions in a given LMWG, and the difference between $G_{\text {crys }}$ and $G_{\text {solv }}$ can be related to the solubility of a given molecule on a given solvent. In the case of a molecule which is not soluble in a given solvent, $G_{c r y s} \ll$ $G_{\text {solv }}$, while for a soluble molecule the difference between these two parameters will be minor.

\section{RESULTS. $\Delta \mathbf{G}_{\text {fiber }}$ CALCULATION}

In order to determine whether a fiber state could represent the thermodynamic minimum, we define the following energy penalty parameters:

$\gamma_{l s}=0$ : (red face exposed at the surface, Fig. 1) Solvophilic faces that are solvent exposed are assumed to be the lowest energy penalty as these represent the most stable interactions (e.g., a carboxylate group in water). Since we calculate differences in Gibbs energy between states, it is set to zero as a reference point.

$\gamma_{k b}=1$ : (black, internal, Fig. 1) The second coefficient, the solvophobic faces buried, is set to 1 as a slightly less stabilizing interaction than solvent exposed solvophilic faces, although still relatively favorable (e.g., aromatic moieties in aqueous solution, which, when buried inside the fiber, can establish $\pi$-stacking interactions).

$\gamma_{k s} \geq 2$ : (black, surface exposed, Fig. 1) The interaction of the solvophobic faces with the solvent is less favorable compared to the two previous types of interactions. This mimics, for example, the exposure of an aliphatic chain to water. The increment of this parameter would be related with increasing the length of the carbon chain.

$\gamma_{l b}>0$ : (red, internal, Fig. 1) There are two possibilities for interactions of a buried solvophilic face:

- $\gamma_{l b} \geq 2$ : solvophilic faces buried in a solvophobic environment - at best comparable to $\gamma_{k s}$ (e.g., a carboxylate group and the repulsion it generates depending on the $\mathrm{pH}$, and hence, in the fraction of charged groups in the inner part of the fiber).

- $0<\gamma_{l b}<2$ : solvophilic faces buried in such a way that they interact favorably. This case takes into account the possibility that solvophilic faces can establish interactions in the buried areas with other solvophilic faces (e.g., through hydrogen bonding of amide groups in aqueous solution, or through $\pi$-stacking interactions between aromatic molecules in organic solvent. The parameter is varied depending on the strength of the intermolecular interactions). Stability expected to be towards that of solvophilic exposed to the solvent $\left(\gamma_{l s}=0\right)$ and always more stable than the solvophobic exposed to the solvent $\left(\gamma_{k s} \geq 2\right)$. 
As the parameters $\gamma_{l s}$ and $\gamma_{k b}$ are set to 0 and 1 respectively for the $G_{\text {fiber }}$ calculations, $G_{\text {crys }}$ only depends on the parameter $\gamma_{l b}$ and $G_{s o l v}$ only on $\gamma_{k s}$, and hence the plots are made with these parameters in the $y$-axes and with the $d$ variable in the $x$ axes. The plots also show the evolution of the optimum $d$, defined as the $d$ value with the minimum $\Delta G_{\text {fiber }}\left(d_{\text {min }}\right)$, in function of the $y$-axes. If the $d_{\min }$ is lower than 30 , which is the maximum $d$ considered in the plots, then the fiber is the thermodynamic favored assembly.

The results show that for the $S q_{0}$ the $\Delta G_{\text {fiber }}$ is positive for all the values of $\gamma_{k s}$ (Fig. $2 \mathrm{~A}$ ) and it gets closer to 0 as $d$ rises (Fig. $2 \mathrm{~A}$ and F). However, by adding amphiphilicity to the units $(n \neq 0)$ the results show values of $\Delta G_{\text {fiber }}$ below 0 and values of $d_{\min }$ below 30 . These evidence the fiber to be the thermodynamic favored product. Differences can be found between fibers which have solvophobic faces exposed to the solvent, $S q_{1}$ (Fig. 2 B-C), and fibers which do not expose solvophobic faces to the solvent, $S q_{2}$ and $S q_{3}$ (Fig. 2 D-E). In addition, results for the four types of $S q$ only as a function of $d$ $\left(\gamma_{l b}\right.$ and $\gamma_{k s}$ are set to 2) are shown for more direct comparison between the four cases (Fig. $2 \mathrm{~F}$ ). It can be seen how $\Delta \mathrm{G}_{\text {fiber }}$ tends to the minimum at high $d$ 's for $S q_{0} ; S q_{1}$ shows a $d_{\text {min }}$ which is different from 2 while $S q_{2}$ and $S q_{3}$, which are overlapped, have a $d_{\min }$ which is 2 (favoring the thinnest possible fiber).
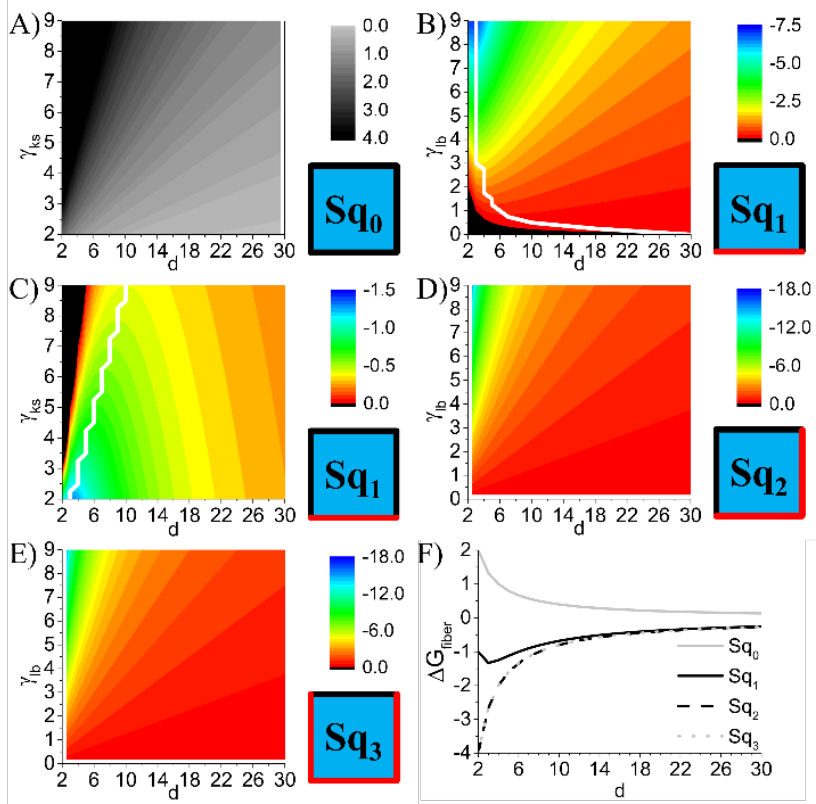

Figure 2. $\Delta G_{f i b e r}$ for $S q_{0}$ (A), $S q_{1}$ (B-C), $S q_{2}$ (D) and $S q_{3}$ (E) as a function of $d$ and: as a function of $\gamma_{k s}$ with fixed $\gamma_{l b}$ (A and C); and as a function of $\gamma_{l b}$ with fixed $\gamma_{k s}$ (B, D and E). $\Delta G_{f i b e r}$ for $S q_{0}$, $S q_{1}, S q_{2}$ and $S q_{3}$ as a function of $d$ with fixed $\gamma_{k s}$ and $\gamma_{l b}$ (both =2) (F). $d_{\min }$ is represented with a white line (A-E).

Fibers exposing both solvophilic and solvophobic faces at the surface have a preferred width ( $d_{\min }$ value) which is dependent on the interaction parameters (Fig. 2 B-C, F). When buried solvophilic faces are able to form interactions between themselves $\left(0<\gamma_{l b}<2\right)$ wider fibers $\left(4<d_{\min }<30\right)$ are also stabilized relative to the crystal state (white line in Fig. 2 B). However, as $\gamma_{l b}$ increases (decreased stability of the solvophilic face when buried) the $d_{\min }$ value rapidly decreases and thin fibers $\left(2<d_{\text {min }}<4\right)$ are obtained (Fig. 2 B). Conversely, as the energy penalty for exposing solvophobic faces to the solvent increases, the fibers get wider (Fig. 2 C) in order to decrease the fraction of corner units (Fig. 1). These results suggest that the width of amphiphilic fibers can be tuned with changes in the unit of LMWG.

The results for the fibers exposing only solvophilic faces show a constant fiber width $\left(d_{\min }\right)$ for $\gamma_{l b} \neq 0$. (Fig.2 D-F). This is not surprising as the minimum fiber width $(d=2)$ shows no buried solvophilic face and, as there is not solvophobic face exposed to the solvent on these fibers, burying solvophilic faces involves the only destabilizing effect. In the Supporting Information it is shown how changing the base of the prism to hexagons (Fig. S3-S4 C, Hx) results in some amphiphilic units not favoring fibers as their thermodynamic minimum, but others do, depending on $n$. Overall, this packing model demonstrates that fibers can be at thermodynamic equilibrium under some conditions due to the amphiphilicity of the LMWG.

\section{COMPARISON WITH EXPERIMENTAL OBSERVATIONS FOR KNOWN LMWG}

The model has successfully demonstrated that $1 \mathrm{D}$ packing can represent the thermodynamic minimum. This is demonstrated by using $S q$ (cubes) and a packing which increases equally in all the directions of the $x y$-plane in order to provide a situation where both $1 \mathrm{D}$ and $3 \mathrm{D}$ packing are possible. The formulation can be extended to other shapes and dimensions and similar results are obtained (see Supporting Information). This suggests that the formulation is flexible and can be adapted to represent specific LMWGs.

When considering a specific example, it is important to consider what interactions will be competing and the relative strength of these interactions. This is a user-input aspect of the model, the model itself does not prescribe the relative weights of the different interactions, rather it evaluates the resulting stability of structures based on the user inputs. As such, the model is able to predict if a self-assembling molecule has parameters within the user-defined bounds then it should preferentially form a fiber rather than a crystal (e.g., a hydrophilic interaction on one face that is twice as strong as the possible interactions between the remaining hydrophobic faces results in a fiber). Or alternatively, the model can be used to rationalize the types of structures found by determining what the relative strengths of the interactions are that could result in such a structure.

Tang et al. demonstrated how the structure for N-fluorenyl9-methyloxycarbonyl-dyphenylananine (Fmoc-FF-OH) varies with the $\mathrm{pH}^{51}$ They showed how at high $\mathrm{pH}$ 's the negatively charged Fmoc-FF- ${ }^{-}$remain in solution, but as the $\mathrm{pH}$ decreases and they become protonated they self-assemble into nanostructures, resulting in the formation of a gel. However, when the $\mathrm{pH}$ is below 6 , the system precipitates due to lateral aggregation (Fig. $3 \mathrm{~F}$ ). Note that Adams et al. reported a single apparent $\mathrm{pKa}$ for this same system at 8.9, observed upon lowering of the $\mathrm{pH}$ using a hydrolyzing sugar ester (gluconolactone). ${ }^{53}$ However, in the Tang report a heat-cool cycle was used at each incremental $\mathrm{pH}$ change in an effort to unlock any kinetic aggregates, and therefore is more likely to represent the equilibrium state. ${ }^{51}$

Prisms with a hexagonal base were used to mimic Fmoc-FF$\mathrm{OH}$ either with one solvophilic face, which represents the $\mathrm{COOH}$ terminus $\left(H x_{1}\right.$, Fig. $\left.3 \mathrm{~A}\right)$, or with two, non-contiguous, solvophilic faces $\left(\mathrm{Hx}_{1+1}\right.$, Fig. $\left.3 \mathrm{C}\right)$, representing the $\mathrm{COOH}$ 
and hydrophilic carbamate $(-\mathrm{CO}(\mathrm{C}=\mathrm{O})-)$ linker. The prism used to represent a certain $\mathrm{LMWG}$ within the model is also a user-input aspect. Due to the simplicity of the model, more than only one shape could be appropriate to represent a given LMWG. Equally, different numbers of solvophilic and solvophobic faces could be used, as is the case for the example of Fmoc-FF-OH. As it is not possible to standardize the way these parameters are decided, the different reasonable possibilities need to be evaluated.

The $\mathrm{pH}$ changes are mimicked by changing the energetic penalties for each type of face (Fig. $3 \mathrm{E}$ ). The solvophilic faces buried $\left(\gamma_{l b}\right)$ change from being highly unfavorable (9) due to the charge repulsion of $\mathrm{COO}^{-}$to be more favorable due to the possibility of forming hydrogen bonds of $\mathrm{COOH}$ (2). The $\gamma_{l s}$ does not involve a penalty (0) when the molecule is charged but it gets less favorable (3.5) when it is neutral. The term for the solvophobic faces exposed to the solvent $\left(\gamma_{k s}\right)$ is kept constant and unfavorable (4.5) but the term for these faces buried $\left(\gamma_{k b}\right)$ changes from involving the same energy penalty as previous due to the proximity of negatively charged groups (4.5) to involve a minimum penalty due to the neutrality which minimizes the repulsion with the $l$ faces, and to the possibility of $\pi$ stacking interactions with other $k$ faces. The value of 4.5 was chosen as it is the half of the maximum energetic penalty used in this work, 9. The maximum $\gamma_{l s}$ (3.5) was chosen to have a value between the minimum $\gamma_{l b}(2)$ and 4.5. The $x$-axes of the results show only the changes in the parameter $\gamma_{l b}$ (Fig. $3 \mathrm{~B}$ and D).
A)

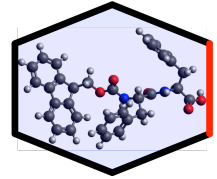

C)

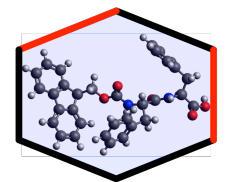

E)

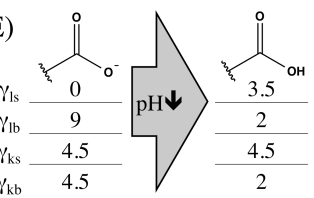

B)

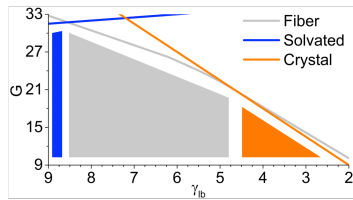

D)
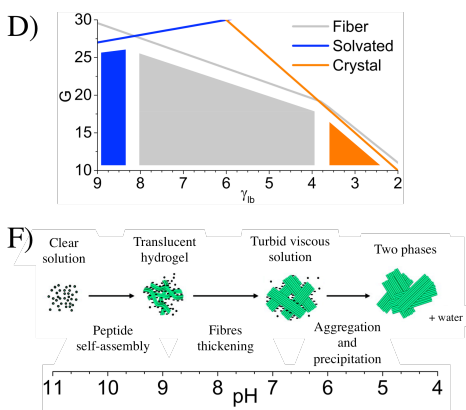

Figure 3. Fitting of the $H x_{1}$ (A) and the $H x_{I+l}$ (C) prisms with the Fmoc-FF-OH molecule (Solvophilic faces in red and solvophobic in black). Results showing the changes of $G_{\text {solv }}$ (Blue), $G_{\text {fiber }}$ (Grey) and $G_{\text {crys }}$ (Orange) for $H x_{1}$ (B) and the $H x_{1+1}$ (D). The colored areas show the most stable state in each region. The plots are presented as a function of $\gamma_{l b}$ but the extreme values for the four parameters to reproduce the non-protonated and protonated states are presented in E. Experimental observation for the system by Tang et al. (F), adapted from ref 51 .

The results for these two prisms are shown as a phase change diagram showing which phase involves the minimum energetic state with colored areas. It has to be taken in consideration that for the $H x_{1+1}$ both solvophilic faces are treated as equal, but only the side representing the acid group realistically suffers significant parameter changes. However, the $H x_{1}$ does not take into account the changes in the interactions with the carbamate group, which although being less important than those in the acid group, they might still be significant. Therefore, the most accurate representation may reasonably be a combination of both hexagonal based prisms. However, although the two prisms differ in the points where the phase transitions occur, both, $H x_{1}$ and $H x_{1+1}$ (Fig. $3 \mathrm{~B}$ and D) show how the system changes from solution to fiber state and finally to the crystal state as the parameters which mimic the $\mathrm{pH}$ drop (Fig. 3 E). Therefore, the model qualitatively reproduces the experimental changes with the $\mathrm{pH}$ shown by Tang et al. (Fig. $3 \mathrm{~F}$ ) involving three different self-assembly states depending on the $\mathrm{pH}$ of the system.

\section{CONCLUSIONS}

A simple packing model for LMWG has been proposed based on prisms with tunable faces with different levels of solvophobicity or solvophilicity. This model includes tunable parameters which can be related to the characteristics of a given gelator, including the shape; the proportion of solvophilic and solvophilic surface; and their solubility and crystallization tendency. For more complex molecular structures, it would become necessary to extend the simple prisms used in this work to form more complex molecular shapes. Nonetheless, the model clearly shows that the amphiphilicity of molecules dictates the tendency to form 1D extended structures versus the $3 \mathrm{D}$ extended crystalline structure and it is shown that by simply changing the strength of interaction parameters (comparable with e.g. introducing extra H-bonding or stacking interactions to favor or disfavor solvent interactions) a system can be switched from preferential 1D assembly to preferential crystallization. Thus, the model demonstrates that for selected classes of LMWGs the fiber structure can be the thermodynamic minimum.

The formulation has also been successfully applied for other regular shapes, which allows to easily fit LMWGs into the model, and also for irregular cross-sections which allows the model to be extended to 2D objects (Fig. S6-S7 and Table S6).

The versatility of the model in terms of shapes and crosssections has allowed the application of the model to reproduce experimental results for a specific LMWG. For example, the $\mathrm{pH}$ responsive gelation of Fmoc-FF-OH, which is soluble at high $\mathrm{pH}$, results in gelation at intermediate $\mathrm{pH}$ and gives rise to lateral aggregation and eventual precipitation at low $\mathrm{pH}$, can be accurately described by simply changing the surface interaction parameter from unfavorable (repulsion of anionic groups at high $\mathrm{pH}$ ) to favorable (fibril formation at intermediate $\mathrm{pH}$ where surface ionization occurs) to unfavorable (low $\mathrm{pH})$. This demonstrates the potential applications of a simple model to enhance the understanding of the thermodynamics of nanostructures formation.

\section{ASSOCIATED CONTENT}

Further formulation to other shapes (Fig. S1-S5 and Table S4-S5), which allow an easier fitting to different real LMWG, and dimensions (Fig. S6-S7 and Table S6). This material is available free of charge via the Internet at http://pubs.acs.org.

\section{AUTHOR INFORMATION}

\section{Corresponding Author}

*tell.tuttle@strath.ac.uk, *rein.ulijn@asrc.cuny.edu Notes

The authors declare no competing financial interest. 


\section{ACKNOWLEDGMENT}

The authors gratefully acknowledge the financial support by the EC $7^{\text {th }}$ Framework Programme Marie Curie Actions via the European ITN SMARTNET No. 316656 and EMERgE/ERC No. 258775 .

\section{ABBREVIATIONS}

Crys, crystal; CS, crystal state; GS, gel state; LMWG, low molecular weight gelator; solv, solvation; Sq, squared based prism.

\section{REFERENCES}

1. Estroff, L. A.; Hamilton, A. D. Water Gelation by Small Organic Molecules. Chem. Rev., 2004, 104, 1201-1218.

2. Yan, X.; Zhu, P.; Li, J. Self-assembly and Application of Diphenylalanine-based Nanostructures. Chem. Soc. Rev., 2010, 39, 1877.

3. Smith, A. M.; Ulijn, R. V. Designing Peptide Based Nanomaterials. Chem. Soc. Rev., 2008, 37, 664-675.

4. Mattia, E.; Otto, S. Supramolecular Systems Chemistry. Nature Nanotechnol., 2015, 10, 111-119.

5. Zhao, X.; Pan, F.; Xu, H.; Yaseen, M.; Shan, H.; Hauser, C. A. E.; Zhang, S.; Lu, J. R. Molecular Self-assembly and Applications of Designer Peptide Amphiphiles. Chem. Soc. Rev., 2010, 39, 34803498 .

6. Zelzer, M.; Ulijn, R. V. Next-generation Peptide Nanomaterials: Molecular Networks, Interfaces and Supramolecular Functionality. Chem. Soc. Rev., 2010, 39, 3351-3357.

7. Zhang, S. Fabrication of Novel Biomaterials Through Molecular Self-assembly. Nature Biotechnol., 2003, 21, 1171-1178.

8. Graham, T. Liquid Diffusion Applied to Analysis. Philos. Trans. R. Soc. London, 1861, 183-224.

9. Aida, T.; Meijer, E.; Stupp, S. Functional Supramolecular Polymers. Science, 2012, 335, 813-817.

10. Terech, P.; Weiss, R. G. Low Molecular Mass Gelators of Organic Liquids and the Properties of Their Gels. Chem. Rev., 1997, 97, 3133-3160

11. Adler-Abramovich, L.; Gazit, E. The Physical Properties of Supramolecular Peptide Assemblies: From Building Block Association to Technological Applications. Chem. Soc. Rev., 2014, 43, 6881-6893.

12. Steed, J. W. Anion-tuned Supramolecular Gels: a Natural Evolution from Urea Supramolecular Chemistry. Chem. Soc. Rev., 2010, 39, 3686-3699.

13. Smith, D. K. Lost in Translation? Chirality Effects in the Self-assembly of Nanostructured Gel-phase Materials. Chem. Soc. Rev., 2009, 38, 684-694.

14. Ghadiri, M. R.; Granja, J. R.; Milligan, R. A.; McRee, D. E.; Khazanovich, N. Self-assembling Organic Nanotubes Based on a Cyclic Peptide Architecture. Nature, 1993, 366, 324-327.

15. Hartgerink, J. D.; Benlash, E.; Stupp, S. L. Self-Assembly and Mineralization of Peptide-Amphiphile Nanofibers. Science, 2001, 294, 1684-1688.

16. Raeburn, J.; Adams, D. J. Multicomponent Low Molecular Weight Gelators. Chem. Commun., 2015, 51, 5170-5180.

17. Reches, M.; Gazit, E. Casting Metal Nanowires within Discrete Self-assembled Peptide Nanotubes. Science, 2003, 300, 625627.

18. Frederix, P., W. J. M.; Scott, G. G.; Abul-Haija, Y. M.; Kalafatovic, D.; Pappas, C. G.; Javid, N.; Hunt, N. T.; Ulijn, R. V.; Tuttle, T. Exploring the Sequence Space for (Tri-)peptide Selfassembly to Design and Discover New Hydrogels. Nature Chem., 2015, 7, 30-37.

19. Gronwald, O.; Shinkai, S. Sugar-Integrated Gelators of Organic Solvents. Chem.-Eur. J., 2001, 7, 4328-4334.

20. Reinhoudt, D. New Sugar-based Gelators Bearing ApNitrophenyl Chromophore: Remarkably Large Influence of a Sugar Structure on the Gelation Ability. J. Chem. Soc., Perkin Trans. 2, 1998, 2585-2592.
21. Fleming, S.; Ulijn, R. V. Design of Nanostructures Based on Aromatic Peptide Amphiphiles. Chem. Soc. Rev., 2014, 43, 81508177.

22. Cui, H.; Muraoka, T.; Cheetham, A. G.; Stupp, S. I. SelfAssembly of Giant Peptide Nanobelts. Nano Lett., 2009, 9, 945-951.

23. Aggeli, A.; Bell, M.; Boden, N.; Keen, J. N.; Knowles, P. F.; McLeish, T. C. B.; Pitkeathly, M.; Radford, S. E. Responsive Gels Formed by the Spontaneous Self-assembly of Peptides Into Polymeric [Beta]-Sheet Tapes. Nature, 1997, 386, 259-262.

24. Hughes, M.; Frederix, P. W. J. M.; Raeburn, J.; Birchall, L. S.; Sadownik, J.; Coomer, F. C.; Lin, I. H.; Cussen, E. J.; Hunt, N. T.; Tuttle, T., et al. Sequence/Structure Relationships in Aromatic Dipeptide Hydrogels Formed Under Thermodynamic Control by Enzyme-assisted Self-assembly. Soft Matter, 2012, 8, 5595-5602.

25. Smith, A. M.; Collins, R. F.; Ulijn, R. V.; Blanch, E. Raman Optical Activity of an Achiral Element in a Chiral Environment. J. Raman Spectrosc., 2009, 40, 1093-1095.

26. Mahler, A.; Reches, M.; Rechter, M.; Cohen, S.; Gazit, E. Rigid, Self-Assembled Hydrogel Composed of a Modified Aromatic Dipeptide. Adv. Mater, 2006, 18, 1365-1370.

27. Dudukovic, N. A.; Zukoski, C. F. Evidence for Equilibrium Gels of Valence-limited Particles. Soft Matter, 2014, 10, 7849-7856.

28. de Jong, J. J.; Lucas, L. N.; Kellogg, R. M.; van Esch, J. H.; Feringa, B. L. Reversible Optical Transcription of Supramolecular Chirality Into Molecular Chirality. Science, 2004, 304, 278-281.

29. Hecht, S. Optical Switching of Hierarchical Self-Assembly: Towards "Enlightened" Materials. Small, 2005, 1, 26-29.

30. Boekhoven, J.; Poolman, J. M.; Maity, C.; Li, F.; van der Mee, L.; Minkenberg, C. B.; Mendes, E.; van Esch, J. H.; Eelkema, R. Catalytic Control Over Supramolecular Gel Formation. Nature Chem., 2013, 5, 433-437.

31. Pashuck, E. T.; Stupp, S. I. Direct Observation of Morphological Tranformation from Twisted Ribbons into Helical Ribbons. J. Am. Chem. Soc., 2010, 132, 8819-8821.

32. Korevaar, P. A.; Newcomb, C. J.; Meijer, E.; Stupp, S. I. Pathway Selection in Peptide Amphiphile Assembly. J. Am. Chem. Soc., 2014, 136, 8540-8543.

33. Hirst, A. R.; Roy, S.; Arora, M.; Das, A. K.; Hodson, N.; Murray, P.; Marshall, S.; Javid, N.; Sefcik, J.; Boekhoven, J., et al. Biocatalytic Induction of Supramolecular Order. Nature Chem., 2010, 2, 1089-1094.

34. Tidhar, Y.; Weissman, H.; Wolf, S. G.; Gulino, A.; Rybtchinski, B. Pathway-Dependent Self-Assembly of Perylene Diimide/Peptide Conjugates in Aqueous Medium. Chem.-Eur. J., 2011, 17, 6068-6075.

35. Chen, L.; Revel, S.; Morris, K.; C. Serpell, L.; Adams, D. J. Effect of Molecular Structure on the Properties of Naphthalene-Dipeptide Hydrogelators. Langmuir, 2010, 26, 1346613471.

36. Hanabusa, K.; Matsumoto, M.; Kimura, M.; Kakehi, A.; Shirai, H. Low Molecular Weight Gelators for Organic Fluids: Gelation Using a Family of Cyclo(dipeptide)s. J. Colloid Interface Sci., 2000, 224, 231-244.

37. Adams, D. J.; Morris, K.; Chen, L.; Serpell, L. C.; Bacsa, J.; Day, G. M. The Delicate Balance Between Gelation and Crystallisation: Structural and Computational Investigations. Soft Matter, 2010, 6, 4144-4156.

38. Zhu, P.; Yan, X.; Su, Y.; Yang, Y.; Li, J. Solvent-Induced Structural Transition of Self-Assembled Dipeptide: From Organogels to Microcrystals. Chem.-Eur. J., 2010, 16, 3176-3183.

39. Terech, P. Metastability and Sol Phases: Two Keys for the Future of Molecular Gels? $\uparrow$. Langmuir, 2009, 25, 8370-8372.

40. Moffat, J. R.; Smith, D. K. Metastable Two-component Gel-Exploring the Gel-Crystal Interface. Chem. Commun., 2008, 2248-2250

41. Terech, P.; Sangeetha, N. M.; Maitra, U. Molecular Hydrogels from Bile Acid Analogues with Neutral Side Chains: Network Architectures and Viscoelastic Properties. Junction Zones, Spherulites, and Crystallites: Phenomenological Aspects of the Gel Metastability. J. Phys. Chem. B, 2006, 110, 15224-15233. 
42. Wang, Y.; Tang, L.; Yu, J. Investigation of Spontaneous Transition from Low-molecular-weight Hydrogel into Macroscopic Crystals. Cryst. Growth Des., 2008, 8, 884-889.

43. De Rudder, J.; Bergé, B.; Berghmans, H. Competition Between Gelation and Crystallization in Solutions of Syndiotactic Polystyrene in cis-Decalin. Macromol. Chem. Phys., 2002, 203, 2083 2088.

44. Roy, B.; Bairi, P.; Nandi, A. K. Metastability in a Bicomponent Hydrogel of Thymine and 6-Methyl-1, 3, 5-triazine-2, 4diamine: Ultrasound Induced vs. Thermo Gelation. Soft Matter, 2012, 8, 2366-2369.

45. Houton, K. A.; Morris, K. L.; Chen, L.; Schmidtmann, M.; Jones, J. T. A.; Serpell, L. C.; Lloyd, G. O.; Adams, D. J. On Crystal versus Fiber Formation in Dipeptide Hydrogelator Systems. Langmuir, 2012, 28, 9797-9806.

46. Williams, R. J.; Smith, A. M.; Collins, R.; Hodson, N.; Das, A. K.; Ulijn, R. V. Enzyme-Assisted Self-assembly Under Thermodynamic Control. Nature Nanotechnol., 2009, 4, 19-24.

47. Dixit, N. M.; Zukoski, C. F. Competition Between Crystallization and Gelation: a Local Description. Phys. Rev. E, 2003, 67,061501 .
48. Cheng, S.; Stevens, M. J. Self-assembly of Chiral Tubules. Soft Matter, 2014, 10, 510-518.

49. Hartman, P.; Bennema, P. The Attachment Energy as a Habit Controlling Factor: I. Theoretical Considerations. J. Cryst. Growth, 1980, 49, 145-156.

50. Weissbuch, I.; Popovitz-Biro, R.; Lahav, M.; Leiserowitz, L. Understanding and Control of Nucleation, Growth, Habit, Dissolution and Structure of Two- and Three-Dimensional Crystals Using Tailor-made-auxiliaries. Acta Crystallogr., Sect. B: Struct. Sci., 1995, 51, 115-148.

51. Tang, C.; Smith, A. M.; Collins, R. F.; Ulijn, R. V.; Saiani, A. Fmoc-Diphenylalanine Self-Assembly Mechanism Induces Apparent pKa Shifts. Langmuir, 2009, 25, 9447-9453.

52. Privalov, P. L.; Makhatadze, G. I. Contribution of Hydration to Protein Folding Thermodynamics: II. The Entropy and Gibbs Energy of Hydration. J. Mol. Biol., 1993, 232, 660-679.

53. Raeburn, J.; Pont, G.; Chen, L.; Cesbron, Y.; Levy, R.; Adams, D. J. Fmoc-diphenylalanine Hydrogels: Understanding the Variability in Reported Mechanical Properties. Soft Matter, 2012, 8, 1168-1174.

\section{For Table of Contents (TOC) only:}

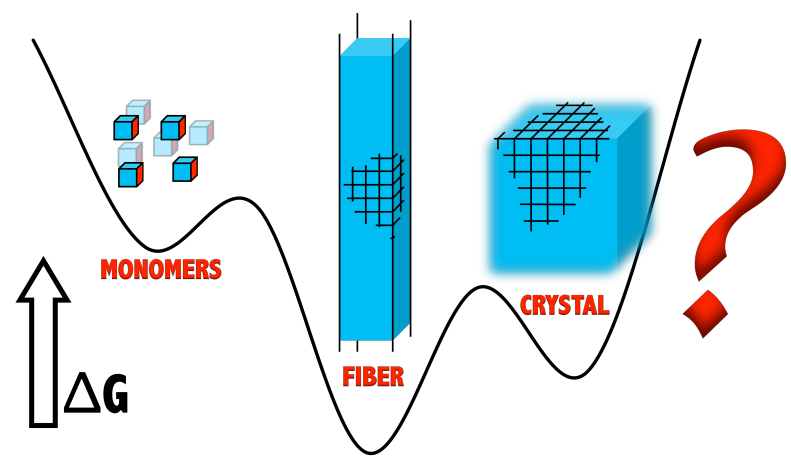

